\title{
Practical Neurology reader survey 2016
}

\section{Geraint N Fuller, ${ }^{1}$ Philip E M Smith ${ }^{2}$}

\begin{abstract}
${ }^{1}$ Department of Neurology, Gloucester Royal Hospital, Gloucester, UK

${ }^{2}$ University Hospital of Wales, Cardiff, UK
\end{abstract}

\section{Correspondence to}

Dr Geraint N Fuller, Department of Neurology, Gloucester Royal Hospital, Gloucester GL1 3NN, UK; geraint.fuller@glos.nhs.uk

Accepted 28 September 2016

CrossMark

To cite: Fuller GN, Smith PEM. Pract Neurol 2016;16:428-430.

\begin{abstract}
The essential point of Practical Neurology is that it is practical in the sense of being useful for everyone who sees neurological patients and who wants to keep up to date, and safe, in managing them. In other words this is a journal for jobbing neurologists who plough through the tension headaches and funny turns week in and week out.
\end{abstract}

\section{INTRODUCTION}

The success of most academic journals can be captured in a single number, its impact factor. This provides a measure of the importance of the research being published and the degree to which it influences other authors in the field. Practical Neurology, in contrast, defines its impact by other measures: how often it is read; how useful are the articles to clinical neurologists; how often it influences clinicians in the clinic and whether it is interesting, entertaining and thought provoking.

In the spring of this year, we undertook a web-based questionnaire to find out how well the journal serves its readers, looking for your comments and suggestions. BMJ publishing conducted the survey using a format developed for all their journals, but with flexibility to allow us to ask questions particularly relevant to Practical Neurology.

\section{RESULTS}

There were 293 responses of which $92 \%$ were from the members of the Association of British Neurologists. The responses from various age ranges were: 26-34 years, $15 \%$; 35-54 years, 62\%; 55-64 years, $18 \%$ and $65+$ years, $5 \%$. Most respondents regularly read the print version: $68 \%$ once a month or more; $17 \%$ once a quarter; $14 \%$ less often or online only. Seventeen per cent said they read all of it, $45 \%$ most of it, $30 \%$ a few articles and $7 \%$ did not read the print journal.
We asked readers how often Practical Neurology impacted on their work, research or practice, with $46 \%$ answering 'frequently', 33\% 'sometimes', 20\% 'occasionally' and 1\% 'never' (figure 1). The 92 related free-text comments emphasised the journal's practical aspects; common terms included 'relevant' (14 times) and daily, clinical or current 'practice' (22 times).

Readers recognised the journal as providing reliable information; on a 10-point scale from 'not at all' to 'extremely', 83\% scored over 8. The 169 free-text comments here (figure 2) are divided into four domains: very negative (none), negative, such as 'does not publish clinical trials or original research and has no impact factor' (3 (1.8\%)); neutral, such as 'I believe it is reliable' (13 (7.7\%)); positive, such as 'I find it useful and reflects real-life neurology' (79 $(46.7 \%))$ and very positive, such as 'excellent journal and highly relevant to clinical practice' (74 (43.8\%)).

We also asked readers if they found different article types interesting, useful or relevant. All article types were considered interesting, each getting between 127 and 183 positive responses-'A difficult case' and 'Neurological rarities' scored highest. 'Reviews' and 'How to do it' articles were the most useful (158-193) and relevant (137-175). Unusual case-based articles, such as 'Neurological rarities', 'Clinicopathological conferences' and 'Test yourself' articles were slightly less useful (100-145) and less relevant (7097). Other less formal articles, such as 'Neurological letter from...', 'Me and my neurological illness', 'Carphology' and 'Book club' were less useful (35-40) and less relevant (29-54).

The free-text comments here were very positive: reviews received the most positive comments, followed by case reports of different sorts, and many highlighted the journal's humour and readability. 


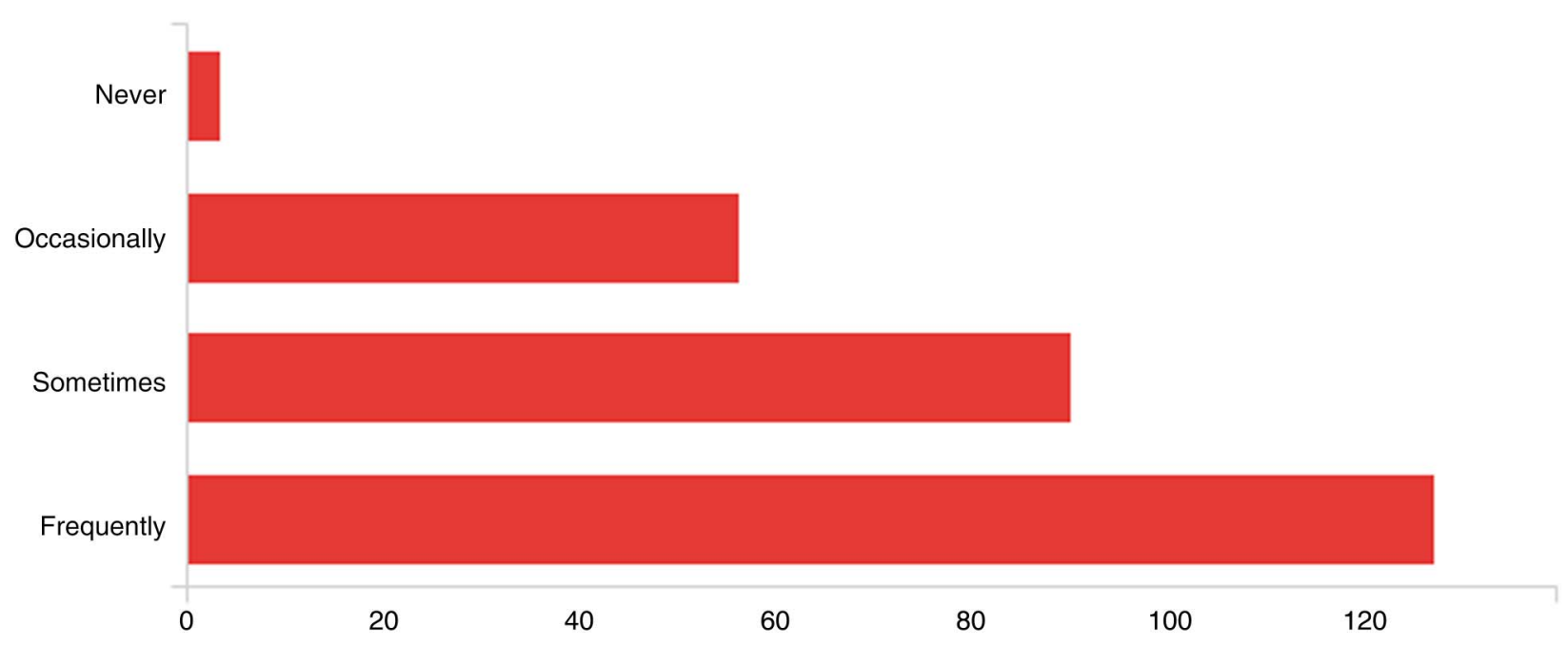

Figure 1 How often has something you read in Practical Neurology impacted on or been reflected in your work, research or practice?

When asked specifically which parts were least enjoyed, the free text again reflected the numerical scores, with over 20 responses saying they liked it all. However, the less formal article types-'the bits and bobs bits' as one respondent put it-were least enjoyed.

The editorials were scored as interesting (165), modestly useful (87) and relevant (85), but did prompt one specific comment: 'the editorial-it's just flannel'.

The survey requested suggestions for new features. Over 100 specific responses to this ranged from specific topics for review, to suggested series (controversies in neurology), to cartoons and a crossword.

\section{COMMENTS}

These results are overall very positive. Over half of the respondents read all or most of the journal regularly and readers valued the information and found it interesting. The more detailed comments suggest that our authors and reviewers are judging their audience well and the articles are pitched at the right level. Unsurprisingly, the most clinically relevant articles - 'Reviews' and 'How to do it' articles-were seen as being most useful and relevant. The less immediately relevant articles, such as the 'Neurological letter from...' and Carphology, were interesting but less useful and relevant. The wide range of imaginative suggestions for future articles reflects our readers' commitment and enthusiasm for the journal.

Acknowledgements We would like to take this opportunity to thank our authors and reviewers for everything they do and to thank everyone who took part in the survey. The survey was conducted by BMJ Publishing.

Competing interests None.

Provenance and peer review Commissioned, internally peer reviewed. 


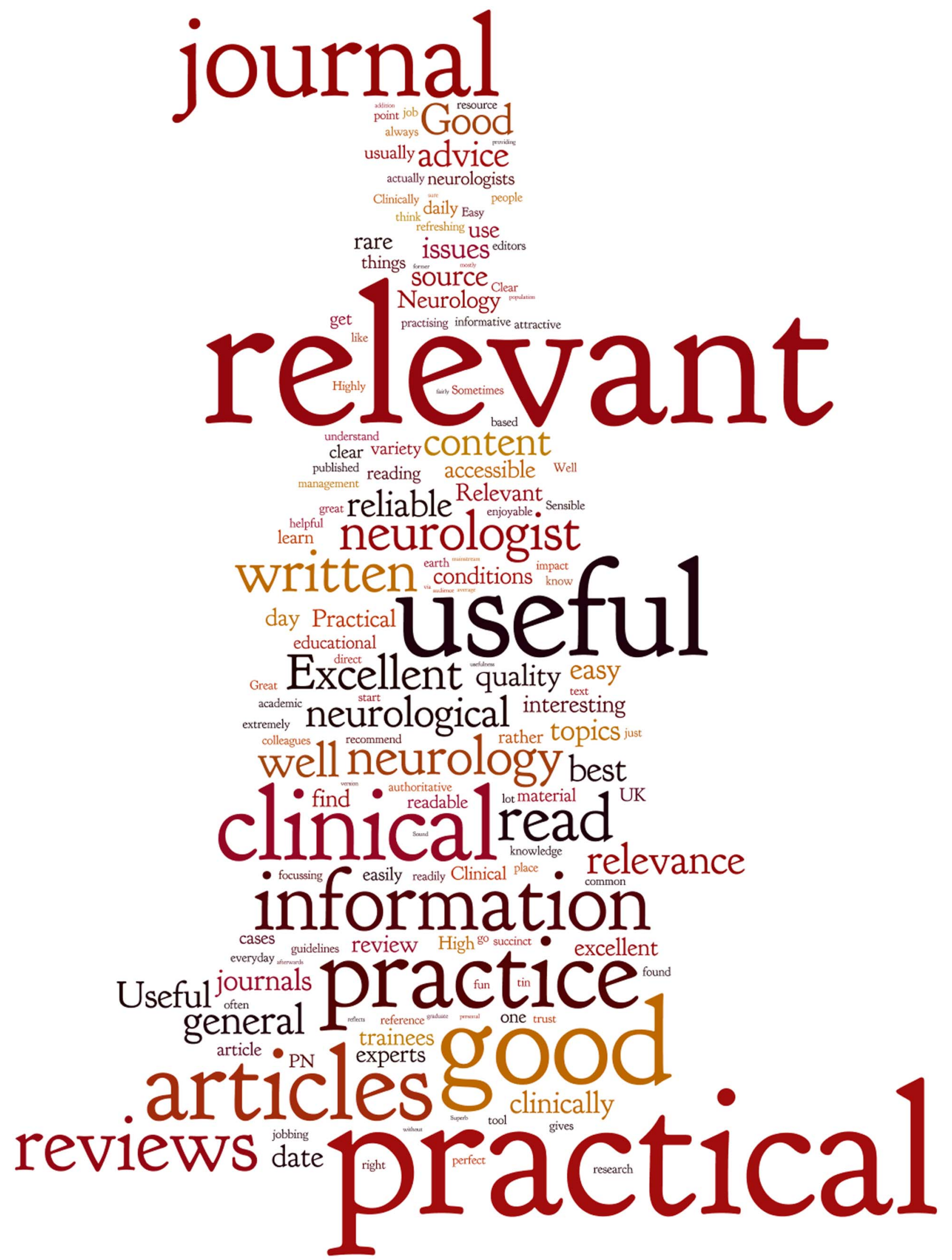

Figure 2 Word cloud generated using Wordle (http://www.wordle.net/create) of 168 responses from readers when asked why they would recommend Practical Neurology. 